\title{
34. The Cometary and Asteroidal Origins of Meteors
}

\author{
ĹUBOR KRESÁK \\ Astronomical Institute of the Slovak Academy of Sciences \\ Bratislava, Czechoslovakia
}

\begin{abstract}
A quantitative examination of the gravitational and nongravitational changes of orbits shows that for larger interplanetary bodies the perturbations by Jupiter strongly predominate over all other effects, which include perturbations by other planets, splitting of comet nuclei and jet effects of cometary ejections. In an approximation to the restricted three-body problem, Sun-Jupiter-comet/asteroid, the value of the Jacobian integral represents a parameter of conspicuous stability which can be applied to delineate the evolutionary paths of the potential parent bodies of the meteoroids in the system of conventional orbital elements. Earth-crossing orbits can be reached along three main paths by the comets, and along two by the asteroids.

The structure of meteor streams, however, indicates that the mutual compensation of the changes in individual elements entering the Jacobian integral, which is characteristic for the comets, does not work among the meteoroids. It appears that additional forces of a different kind must exert appreciable influence on the motion of interplanetary particles of meteoroid size. Nevertheless, the distribution of the Jacobian constant in various samples of meteor orbits, from those of faint Super-Schmidt meteors up to those of meteorite-dropping fireballs, furnishes some information on the type of their parent bodies and on the relative contribution of individual sources.
\end{abstract}

$\mathrm{T}$ HE EVOLUTION OF METEOR ORBITS May be divided into three principal phases: (I) the evolution of the orbit of the parent body, (II) the differential acceleration at the moment of separation, and (III) the subsequent evolution. Under (II) we assume a separation from a sizeable body, with dimensions of the order of $10^{4} \mathrm{~cm}$ and more, for which the dynamical effects of solar radiation and interplanetary magnetic fields are negligible. A progressive fragmentation can obviously continue in smaller particles as well.

The only unquestioned primordial source of meteoroids is comet nuclei. Another plausible but not definitely confirmed alternative source is the asteroids. An essential difference in the possibility of identification of these two sources consists in the distribution of their perihelion distances. Fifty-six percent of known comets have their perihelia situated within the orbit of the Earth; consequently, many meteors can be observed in orbits deviating very little from that of their parent comet, and they are easily related to it. This is practically impossible for normal asteroids, as only 0.2 percent of numbered asteroids come inside the Earth's orbit. Moreover, some or all of these peculiar objects may actually be extinct comet nuclei (Öpik, 1963).

Very important information on the evolution of meteoroids is furnished by meteor showers. The large proportion of meteors associated with showers indicates that the lifetime of individual meteoroids cannot be much longer than the life- 
time of individual meteor streams, which evidently do not vanish through diffusion into the sporadic background. If there is indeed a significant contribution of asteroidal meteors, their ages would hardly be much greater than the ages of cometary meteors since interaction with the Earth's atmosphere does not indicate any striking difference in resistance to disintegration. The low age limit of small bodies reduces the potential significance of phase (III) as compared to phase (I), but only as far as gravitational effects are concerned. At the same time, the observed dispersion of meteor streams sets an upper limit to the effects of phase (II) on shower meteors, but this is still much higher than the expected effect of small ejection velocities.

From the dynamical point of view, the problem of cometary or asteroidal origin is essentially a problem of the aphelion distances of the parent bodies. If the aphelion distance is larger than, or at least approximately equal to, the heliocentric distance of Jupiter, a cometary origin appears beyond doubt even when the comet cannot be identified. If the aphelion is located distinctly inside the orbit of Jupiter, a direct identification of the parent body is generally impossible, and the possible evolutionary paths from the comet or asteroid system must be considered.

One meteor shower, the Geminids, is of particular interest in this respect. Most members of this shower, optically the strongest permanent shower in the northern hemisphere, have their aphelia concentrated within a range of $r$ from 2.5 to 2.7, right in the middle of the asteroid belt. It is absolutely impossible to assume a displacement of this entire stream from a Jupiter-crossing orbit, and therefore an orbit of small aphelion distance must previously have been occupied by the parent body. It appears highly probable that this was a comet; but if this is true one has to explain how the aphelion has escaped from the comet system.

There are several additional streams for which parent comets are unknown, and some of them also have aphelia situated far inside the orbit of Jupiter. These streams are characterized by relatively small perihelion distances ( $\delta$ Aquarids0.08 , Arietids-0.09, Geminids-0.14, ‘ Aquarids $-0.21, \zeta$ Perseids -0.34 ) compared with the streams of known comets (Draconids-0.99,
Leonids -0.98 , Perseids-0.95, Lyrids -0.92 , Ursids- -0.92 , Andromedids -0.86 , Orionids0.58 ). Loosely dispersed streams of known parent comets show intermediate values ( $\alpha$ Capricornids -0.56 , Taurids $-0.34, \beta$ Taurids -0.34 ). This evidence supports the explanation that the absence of a parent comet might be simply a consequence of its early extinction or disintegration in an orbit lying close to the Sun.

A considerable reduction of aphelion distance is a prerequisite of the effective operation of nongravitational forces in the third phase of evolution. For larger particles the gravitational perturbations by Jupiter make this rather difficult. According to Oopik (1951) and Whipple (1955) there is very little chance that particles with diameters exceeding $0.1 \mathrm{~cm}$ can be transported across the Jupiter barrier. However an important object, Comet Encke, proves that comets really can contract their orbits down to $Q=4$.1, at least. Comet Encke is a relatively large object which may have survived many more revolutions in its present orbit than has an average short-period comet. Even so its uniqueness demonstrates that a transition into an orbit of this type is a possible but very rare event, occurring perhaps once in $10^{4}$ years.

\section{THE TISSERAND INVARIANT}

It follows from the general features of the solar system that the major orbital changes of the parent bodies of the meteoroids are due to perturbations by Jupiter. Approximating Jupiter's action by the restricted three-body problem, we can use a function of the elements of maximum stability, the simplified Jacobian integral or Tisserand invariant,

$$
T=a^{-1}+2 a_{j}^{-3 / 2} a^{1 / 2}\left(1-e^{2}\right)^{1 / 2} \cos i
$$

and consider the evolutionary paths along which this function maintains its constant value. Where the inclination remains low, $\cos i \simeq 1$, leaving a function of two variables

$$
T_{0}=a^{-1}+2 a_{j}^{-3 / 2} a^{1 / 2}\left(1-e^{2}\right)^{1 / 2}
$$

which is almost constant.

An analysis of available long-term integration of cometary orbits (Narin and Pierce, 1964; Kazimirchak-Polonskaya, 1967; Belyaev, 1967; 
Marsden, 1968, 1969a, 1970a, 1970c; Everhart and Raghavan, 1970; etc.) enables us to obtain a general insight into the degree of stability of $T$ and $T_{0}$, and on the relative importance of different sources of variations in these quantities.

Most serious is the effect of the eccentricity of Jupiter's orbit, neglected in the restricted threebody problem. This produces mean absolute changes in $T$ of \pm 0.001 after approaches to within $0.3 \mathrm{AU}$ and \pm 0.005 after approaches to $0.1 \mathrm{AU}$. The former case occurs, for an average short-period comet, once in about 200 years, the latter case once in about 1000 years. During a total integration time of about 10000 years, no change exceeding \pm 0.028 has been recorded. The mean cumulative effect can be estimated at \pm 0.001 per century, with 2 to 3 percent probability of a change exceeding \pm 0.010 per century. The changes due to perturbations by other major planets are generally smaller than the effect of Jupiter's eccentricity, the extreme value recorded being -0.007 after an approach to within $0.2 \mathrm{AU}$ of Saturn. This obviously cannot happen to a comet of the Jupiter family.

The splitting of comet nuclei is found to introduce changes of the order of $\pm 10^{-5}$ to $10^{-4}$; but after the tidal disruption of Sun-grazing comets changes of \pm 0.001 to 0.002 have been observed. The present secular acceleration or deceleration of periodic comets by jet effects (Marsden, 1968) introduces changes of \pm 0.001 in $10^{3}$ to $10^{4}$ years, with the extreme of +0.001 per 300 years for $\mathrm{P} /$ Honda-Mrkos-Pajdusáková and $\mathrm{P} /$ Encke. It is obviously possible that a rapid mass loss, especially for comets of very small perihelion distance, can make the changes appreciably higher and progressive.

The variations in $T_{0}$ for short-period comets, are, on the average, twice as large as those in $T$. The variations in $a^{-1}$, the Jacobian constant in the two-body problem or in the three-body problem with a fixed coordinate system, are as much as 20 times as large. No checks were attempted for the asteroids, but it is evident that in this case some of the above effects are absent, and the remaining ones much smaller than for the comets.

These quantitative estimates suggest that a crossing of the Jupiter barrier, and the subsequent transition into an orbit of the Geminid or Apollo type, is possible only if the original value of $T$ is

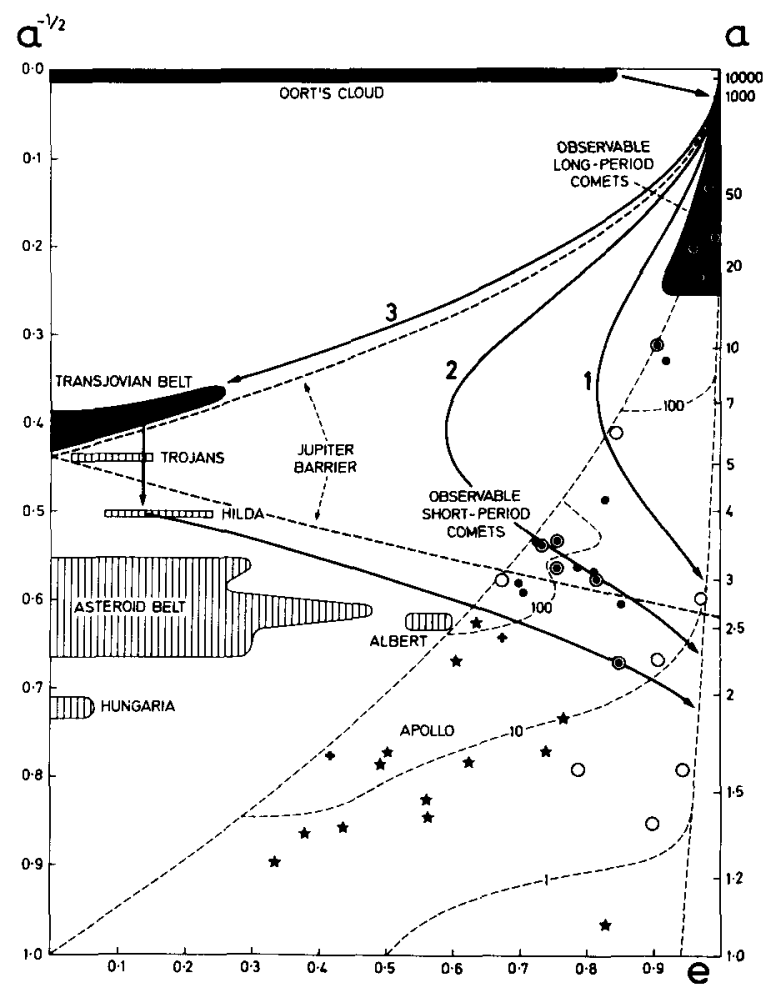

Figure 1.-Potential evolutionary paths of comets into short-period orbits. Heavy line (1) for $T_{0}=0.40$; (2) for $T_{0}=0.50 ;(3)$ for $T_{0}=0.585$. Individual known objects are indicated only if the Earth-crossing condition $q<1$ (dashed curve close to the diagonal) is satisfied. Black dots are short-period comets; circles, meteor streams; stars, Apollo asteroids; crosses, meteorites. Thin dashed lines within the limits $q=0.06$ (curve at extreme right) and $q=1.00$ indicate the corrected relative abundances, 1, 10, and 100, of Super-Schmidt meteors for different combinations of $a^{-1 / 2}$ and $e$ (Kresák, 1967).

near 0.58 . The limitations of this process can be shown in a diagram with paths of constant $T_{0}$ plotted as a function of semimajor axis and eccentricity (fig. 1). A comet starting from Oort's cloud could be observed from the Earth if the combined stellar and planetary perturbations significantly reduced its perihelion distance. Since $T_{0}$ is always smaller than $0.25 q^{1 / 2}$ for $P>800 \mathrm{yr}$, and $T$ is always smaller than $T_{0}$, we can observe only long-period comets with the Jacobian constant far below the critical value of 0.58 , for which $q>6$. From this source long-period meteor streams of random inclination are derived, such as the Lyrids (Comet 1861 I), Perseids (Comet 1862 
III), Orionids and Aquarids ( $\mathrm{P} /$ Halley), and Leonids (P/Tempel-Tuttle).

The capture of long-period comets, presumably with $q=3$ to 5 , into Jupiter's family is characterized by $T_{0}=0.40$ to 0.50 (curves 1 and 2 in fig. 1) and low inclination. The aphelia of these comets concentrate near the Jupiter barrier and are not allowed to recede far from it toward the Sun. Examples of this type among meteor streams are the Andromedids (P/Biela), Draconids (P/Giacobini-Zinner), $\eta$ Ursids (P/PonsWinnecke), Bootids (P/Schwassmann-Wachmann 3 ), and $\alpha$ Capricornids (P/Honda-MrkosPajdušáková). It may be noted that these streams tend to produce temporary displays of short duration, whereas the major long-period showers are of annual appearance and longer duration. Differences in total mass, geocentric velocity and strength of perturbations by Jupiter may be held responsible for this statistical distinction, without the necessity of assuming any differences in origin. The Ursids (P/Tuttle) represent a transition between the two groups.

The concentration of sporadic meteors in the two principal ranges of comet orbits is clearly recognized in every systematic meteor survey, thus presenting a satisfactory evidence of the cometary origin of these objects. On the other hand, a sharp cut-off of aphelia at the Jupiter barrier is missing, and the cisjovian orbits of some meteors have no counterpart in the system of comets. The only gap through which the aphelia can pass the barrier is situated at the highest eccentricities and smallest perihelion distances. These characteristics, best represented by the $\delta$ Aquarids and Arietids, would induce a rapid mass loss, and a comet in such an extreme situation would not survive long. Even the meteoroids following its orbit would be relatively short-lived, as confirmed by the sharp cut-off in the distribution of perihelion distances of meteors at about $q=0.06$, and by some physical peculiarities of meteors just above this limit (Kresák, 1968).

\section{EVOLUTIONARY PATHS}

The most promising evolutionary path (labeled 3 in fig. $1, T_{0}=0.585$ ) leads along the outer edge of the Jupiter barrier. It requires first a capture from a low-inclination orbit, approaching Jupiter from outside ( $q \simeq 6$ ), into a low-eccentricity orbit between Jupiter and Saturn. Accretion of comets in the solar nebula near the present orbits of Uranus and Neptune, with initial low-eccentricity orbits and some spiraling inward (Whipple, 1973) would make this step unnecessary. The latter alternative appears much more plausible from the dynamical point of view, however the long storage of comet-like objects at relatively small distances from the Sun may pose additional problems. In spite of the basic difference between these two processes, the ambiguity is rather irrelevant during the last phase of evolution, since we have satisfactory evidence that a Transjovian belt of comets does exist at present (Kresák, 1972). Members of this belt of comets are generally unobservable from the Earth, with the exception of the abnormally bright and active Comet Schwassmann-Wachmann 1. Nevertheless, long-term integrations indicate that at least three other comets (P/Oterma, $\mathrm{P} /$ Whipple and $\mathrm{P} /$ Shajn-Schaldach, the latter two probably being separated components of one primordial comet) have been captured from this belt since 1850 , and one ( $\mathrm{P} /$ Oterma) was ejected back into the belt. The frequency of documented events of this type is large compared with but one capture from a long-period orbit into the Jupiter family during the same period (P/Kearns-Kwee).

During close approaches to Jupiter, which are obviously of low relative velocity and long duration, members of the Transjovian belt can make a double crossing of the Jupiter barrier, from $q=a(1-e)>a_{j}$ to $Q=a(1+e)<a_{j}$. Subsequent evolution may permit them to change into orbits of the type of Comet Encke. A favorable interplay of perturbations is necessary, with several decelerating approaches to Jupiter, but the process is possible. An approximate $3 / 2$ resonance with Jupiter immediately after crossing of the barrier may trigger this trend of evolution if the initial mean motion is equal to, or slightly above, the exact resonance value. In its low-eccentricity stage the path touches the region of the Hilda asteroids. If any of them should happen to escape from the librating motion (according to Schubart, 1968, two of 21 numbered Hilda asteroids are not librating, but these are just objects of low eccentricity and small aphelion distance), it might evolve in a similar manner.

Non-gravitational effects would begin to act 
much more effectively on an orbit situated entirely within the orbit of Jupiter; this refers not only to the jet effects of escaping matter on live comet nuclei but also the sunward drift of solid particles separated from them. The concentration of sporadic meteor orbits around $e \simeq 0.65, a \simeq 2.8$, $Q \simeq 4.6$, adjoining the concentration of the Jupiter family around $e \simeq 0.70, a \simeq 3.3, Q \simeq 5.6$, may be associated with this evolutionary path.

Also, not far from this area the Albert group of the asteroid belt comes closest to the Earthcrossing limit $q=1$. A definite displacement of the maximum concentration of the bright Prairie Network fireballs, including also the Príbram Meteorite, relative to that of the faint SuperSchmidt meteors (McCrosky, 1968) suggests than many larger meteoroids may have originated from asteroidal collisions. It may be noted that the size dependence of the drag effects of solar radiation would require just the reverse arrangement of orbits, with smaller particles situated inside the orbits of the larger ones.

Similar drag effects would also transport asteroidal meteoroids, produced by low-velocity collisions, from the main belt through the zodiacal cloud to the Sun. Since these effects tend to reduce the original eccentricities, most of the particles would reach the Earth with $e<0.10$, and only a very small fraction with $e>0.25$. This condition restricts the corresponding region of occurence of asteroidal meteors to the lower left edge of the diagram, and the Cyclid stream (Southworth and Hawkins, 1963) can be possibly a result of this process. The concentration of Apollo asteroids around $e \simeq 0.40, a \simeq 1.5, Q \simeq 2.1$, including Eros as the largest representative with $q>1$, and the Lost City Meteorite, may be possibly related to the capture process of Mars investigated by Öpik (1963). The high-eccentricity asteroids of the type of Icarus or Apollo, and the unknown parent bodies of the Geminid, Arietid and other streams, may be extinct comets which may have experienced a similar evolution to what Comet Encke is now undergoing.

\section{DISTRIBUTIONS IN THE JACOBIAN CONSTANT}

Some insight into the relative contribution of meteors of different origin can be obtained by
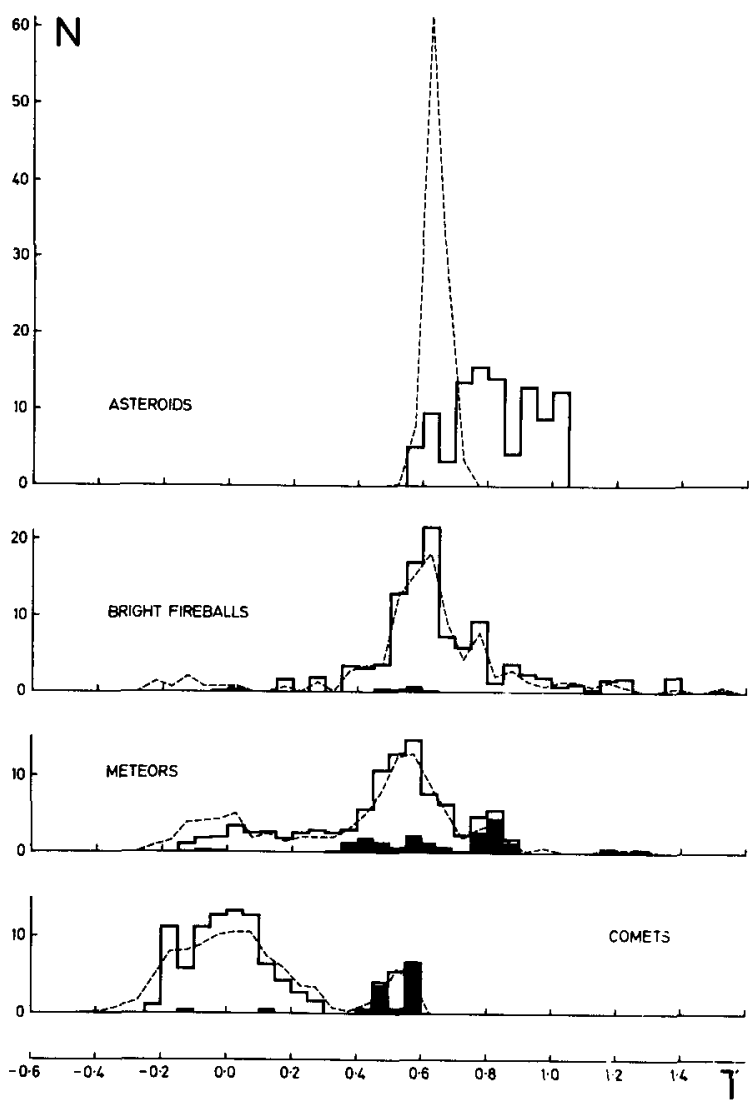

Figure 2.-Normalized distributions of different types of objects in the Jacobian constant $T$. Vertical scale, percentage for $\Delta T=0.05$. Broken lines, observed distribution; histograms, corrected distribution; black areas, contribution of shower meteors or comets associated with them.

comparing the distribution of meteor orbits in $T$ with that of their potential parent bodies. This is done in figure 2 for the asteroids, bright fireballs, faint meteors and comets. Percentages of $T$ within ranges of special interest are listed in tables 1 and 2; for each type of object both the observed and corrected values are given.

\section{The Asteroids}

The observed distribution is taken from the list of 1746 numbered asteroids (Chebotarev, 1970). The corrected distribution includes 13 known asteroidal objects with $q<1$, both numbered and unnumbered (Chebotarev, 1970; Marsden, 1969b, 1970c, 1971; Van Houten et al., 
TaBle 1.-Observed Abundance in Different Ranges of T (Percentages)

\begin{tabular}{|c|c|c|c|c|c|}
\hline $\begin{array}{l}\text { Jacobian constant } T \\
\text { characteristic objects }\end{array}$ & $\begin{array}{c}<0.30 \\
\text { long-period } \\
\text { comets }\end{array}$ & $\begin{array}{c}0.30-0.55 \\
\text { short-period } \\
\text { comets }\end{array}$ & $\begin{array}{c}0.55-0.60 \\
\text { transitional } \\
\text { zone }\end{array}$ & $\begin{array}{c}0.60-0.75 \\
\text { normal } \\
\text { asteroids }\end{array}$ & $\begin{array}{c}>0.75 \\
\text { Apollo } \\
\text { asteroids }\end{array}$ \\
\hline $\begin{array}{l}\text { Asteroids } \\
\text { Comets } \\
\text { Sporadic meteors: } \\
\text { Super-Schmidt meteors } \\
\text { Bright Super-Schmidt meteors } \\
\text { Small camera meteors } \\
\text { Bright fireballs } \\
\text { Brightest fireballs, } H_{E}>30 \mathrm{~km} \\
\text { Brightest fireballs, } H_{E}<30 \mathrm{~km} \\
\text { Meteorites } \\
\text { Shower meteors: } \\
\text { Super-Schmidt meteors } \\
\text { Bright Super-Schmidt meteors } \\
\text { Small camera meteors } \\
\text { Bright fireballs }\end{array}$ & $\begin{array}{r}-\overline{86} \\
29 \\
32 \\
33 \\
7 \\
12 \\
- \\
- \\
34 \\
20 \\
40 \\
38\end{array}$ & $\begin{array}{l}0.3 \\
10 \\
24 \\
30 \\
37 \\
22 \\
38 \\
- \\
- \\
18 \\
30 \\
10 \\
25\end{array}$ & $\begin{array}{r}8 \\
4 \\
11 \\
13 \\
11 \\
15 \\
12 \\
- \\
- \\
7 \\
13 \\
10 \\
25\end{array}$ & $\begin{array}{r}91 \\
- \\
17 \\
17 \\
17 \\
33 \\
19 \\
67 \\
50 \\
\\
8 \\
14 \\
9 \\
12\end{array}$ & $\begin{array}{r}0.2 \\
- \\
19 \\
8 \\
2 \\
23 \\
19 \\
33 \\
50 \\
33 \\
23 \\
31 \\
-\end{array}$ \\
\hline Meteor showers included & \begin{tabular}{|l} 
Leonids \\
Orionids \\
Perseids \\
Lyrids
\end{tabular} & $\begin{array}{l}\text { Quadrantids } \\
\delta \text { Aquarids } \\
\text { Draconids } \\
\text { ‘ Aquarids }\end{array}$ & $\begin{array}{l}\alpha \text { Capricornids } \\
\text { N. Taurids }\end{array}$ & S. Taurids ${ }^{\mathrm{a}}$ & Geminids \\
\hline
\end{tabular}

a Some shower meteors also in adjacent ranges of $T$.

1970). To account for the observational selection, this sample was corrected by a weighting factor

$$
\begin{aligned}
f_{1}= & {\left[2-a^{-1}-a\left(1-e^{2}\right)\right]^{1 / 4} } \\
& \times\left[3-a^{-1}-2 a^{1 / 2}\left(1-e^{2}\right)^{1 / 2} \cos i\right]^{-1 / 4} \sin ^{1 / 2} i
\end{aligned}
$$

representing a square root of the inverse probability of impact per revolution according to Öpik (1951), in relative units. The square root was chosen as a reasonable compromise between the discovery probability of the smallest asteroids detected at very close approaches to the Earth (Apollo, Adonis, Hermes) and that of larger bodies detected at greater geocentric distances. The difference between the observed and corrected distribution is striking indeed, and displaces the values much farther from the critical limit $T=0.58$ into the asteroid region. On the other hand, we do not know how many, and which, Apollo objects are original asteroids.

\section{The Comets}

A total of 607 orbits of different comets (Porter, 1961 ; Marsden, 1966, 1970c) was used to find the observed distribution in $T$. The corrected dis- tribution was determined from 220 returns of 162 different comets with $q<1$, observed during the last hundred years (1871 to 1971). It was attempted to make the resulting data directly comparable with meteor statistics corrected by Whipple's (1954) cosmic weight factors, involving the reciprocal probability of encounter per revolution and the magnitude-velocity dependence. All returns during which periodic comets should have been recovered, even without prediction, were counted. After comparing the distribution of peak apparent magnitudes for independent and predicted discoveries of the last hundred years, the following form of weighting factors was tentatively adopted:

$$
\begin{array}{ll}
f_{2}=1 & \text { for } m \leq m_{0} \\
f_{2}=0.4^{m-m_{0}} & \text { for } m>m_{0} \\
f_{2}=0 & \text { for no recovery }
\end{array}
$$

Here $m_{0}$ denotes the maximum apparent magnitude of the comet during the discovery apparition, or the average from all apparitions during which the comet was located without prediction; $m$ denotes the maximum apparent magnitude during the predicted return in question. The values 
TABLE 2.-Corrected Abundance in Different Ranges of T (Percentages)

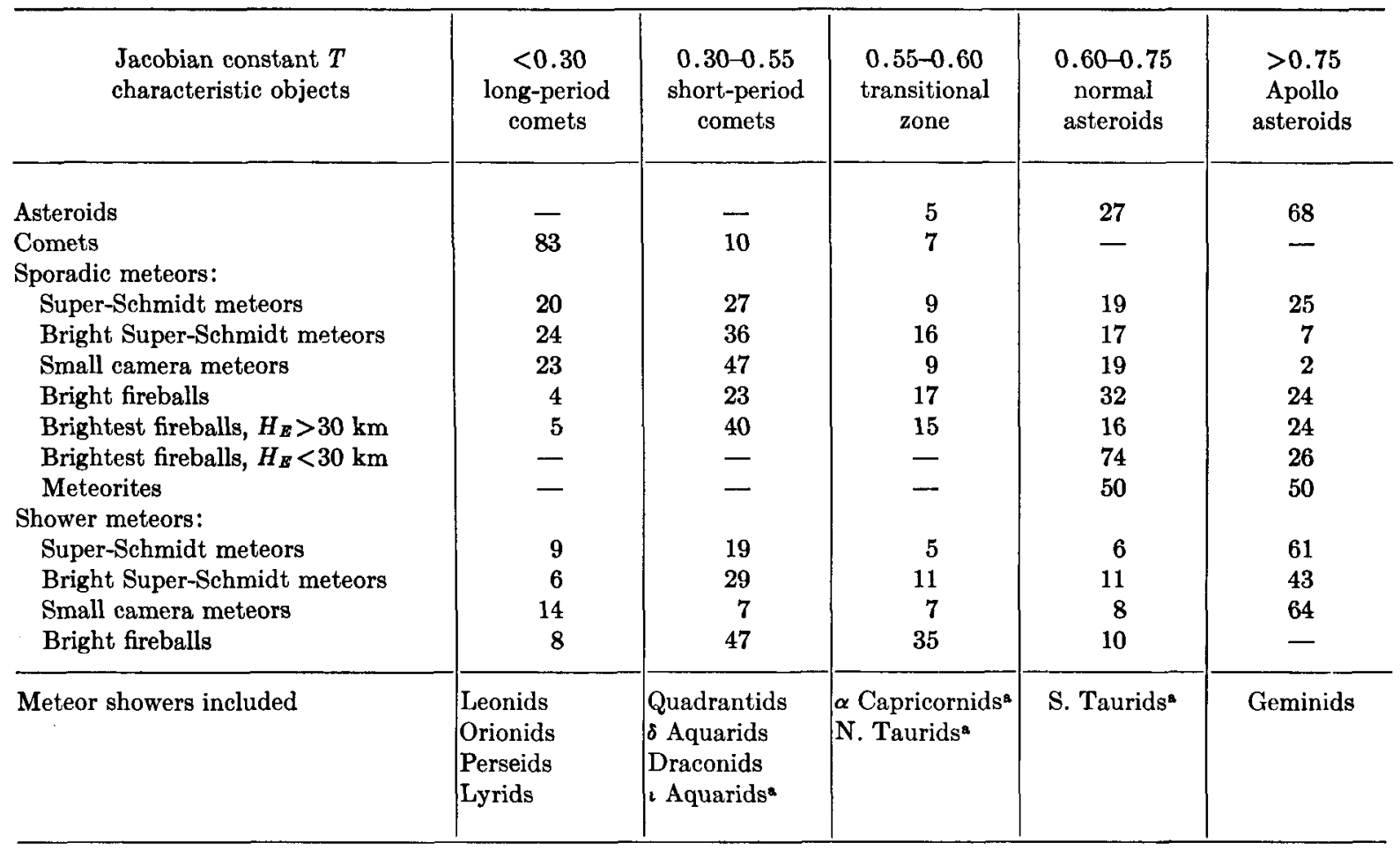

- Some shower meteors also in the adjacent ranges of $T$.

of $m_{0}$ and $m$ were extracted from Vsekhsvyatsky's (1958) summaries of observations up to 1955, and from various sources (e.g., Vsekhsvyatsky's Supplements, Porter's and Marsden's Annual Reports for the R.A.S.) for the last fifteen years. By this procedure, for example, a total weight of 12.9 was assigned to 29 observed returns and one missed return of $\mathrm{P} /$ Encke, a total weight of 6.5 to 10 observed and 10 missed returns of $\mathrm{P} /$ GriggSkjellerup, etc. Only the returns with $q<1$ were taken into account, e.g., prior to 1918 for $\mathrm{P} /$ PonsWinnecke or prior to 1910 for P/Finlay. A separate solution was made for those comets for which associated meteor showers are known.

\section{Bright Fireballs}

The orbits are taken from a list of 141 Prairie Network meteors (McCrosky, 1968, 1970). The low proportion of shower meteors (black areas in fig. 2) is partly due to an intentional selection of sporadic meteors for reduction, and partly to an instrumental limitation by minimum duration. The correction factor reducing the observed numbers to the relative numbers of meteors colliding with the Earth per revolution, is Whipple's (1954) cosmic weight,

$$
f_{3}=V_{G} V_{\infty}{ }^{-4}\left[2-a^{-1}-a\left(1-e^{2}\right)\right]^{1 / 2} \sin i
$$

where $V_{G}$ and $V_{\infty}$ are the geocentric and noatmosphere velocity, respectively. In contrast to the asteroids, the changes introduced by the corrections are relatively insignificant, except for a reduction of the contribution of high-velocity meteors associated with long-period comets. In the corresponding range of negative and small positive values of $T$, the effects of instrumental selection make the frequency rather indeterminate and seriously underestimated (Kresák, 1970). Even so the main feature of the distribution is a pronounced maximum at $T=0.55$ to 0.65 and $Q=3.5$ to 4.5 , in a region adjacent to the Albert group of asteroids.

\section{Faint Meteors}

The orbits were taken from the list of 413 best measurable Super-Schmidt meteors (Jacchia and Whipple, 1961). Some selection effects, especially a preference for meteors of longer duration, are 
involved. However, there is no substantial difference in the distribution of orbital elements compared with the complete list of the orbits of lower accuracy (McCrosky and Posen, 1961). Cosmic weight was introduced by relation (5), as in the preceding case, and the difference between the corrected and observed distribution in $T$ was again found rather insignificant. In spite of a gross agreement, definite distinctions between faint meteors and bright fireballs can be recognized. As already pointed out by McCrosky (1968), the aphelia of Super-Schmidt meteors tend to concentrate farther from the Sun, approximately at the distance of Jupiter. This moderate difference is sufficient to shift the maximum occurrence of $T$ from asteroidal values (above 0.58 ) to cometary ones (below 0.58 ). In the $a / e$ diagram (fig. 1) this means a displacement from the neighborhood of the Albert asteroids to the evolutionary path via the Transjovian belt and to the Jupiter family. The long-period cometary contribution of $T<0.30$ is more pronounced for the faint meteors, the short-period Apollo-type contribution of $T>0.75$ for the bright fireballs, but these differences are affected by observational selection.

Observed and corrected numbers of meteors within five ranges of Jacobian constant $T$ are intercompared in tables 1 to 3 , where two additional meteor samples, 144 Harvard small-camera meteors (Whipple, 1954) and 359 randomly selected faint Super-Schmidt meteors (Hawkins and Southworth, 1961) are included. Two groups of the absolutely brightest fireballs (McCrosky, 1970; Ceplecha, 1970), selected according to the end heights, and two meteorites, Přibram and Lost City, are also added.

Two significant conclusions regarding meteor streams may be drawn from these data. First, meteor showers, in particular the Geminids, occur even in the range of a high asteroidal Jacobian constant, which obviously does not necessarily mean an asteroidal origin. Second, the dispersion in $T$ within meteor streams is surprisingly large compared with the expected variations due to all perturbing effects considered in this paper. The mean deviations within individual showers range between \pm 0.010 (Perseids, Quadrantids, Orionids) and \pm 0.050 (Taurids, Aquarids). This dispersion is definitely not due to measuring errors, and is consistent with the observed radiant scatter which does not involve the inevitable errors in velocity determination. It may be noted that the mean differential velocities within most of the major showers range between 0.3 and $1.5 \mathrm{~km} / \mathrm{s}$, and that the relative velocities of the northern and southern componerts of the twin ecliptical streams (Taurids, $\delta$ Aquarids, ‘ Aquarids) are as high as 4 to $12 \mathrm{~km} / \mathrm{s}$ (Kresák and Porubčan, 1970). In contrast to this, the separation velocities of persistent components of split cometary nuclei range between 0.002 and 0.04 $\mathrm{km} / \mathrm{s}$ (Stefanik, 1966), and ejection velocities of small solid particles computed for the icy-conglomerate model (Whipple, 1951) are of the same order of magnitude. It is also interesting to note that even the dispersion of Jacobian constants within the asteroid families is one order of magnitude smaller than within compact meteor streams, ranging from \pm 0.0006 for the Eos family to \pm 0.006 for the Phocaea family.

It is essential that the mutual compensation of the two terms of equation (1), characteristic for perturbations by Jupiter and very clearly born out by the comets, is entirely absent in meteor streams. While the perturbational changes in $T$ (the Jacobian integral in the rotating coordinate system of the restricted three-body problem) remain about 20 times smaller than those in $a^{-1}$ (the Jacobian integral in the two-body problem) for the comets, the dispersion in both of these quantities is essentially equal in meteor streams. While the orbits of individual members of a stream appear much more alike than the orbits of one heavily perturbed comet at different epochs, the differences in the Jacobian constant are much greater in the former case.

This contradiction suggests that the evolution of meteor orbits cannot be satisfactorily interpreted in terms of gravitational effects alone. An identification of the additional forces with the drag effects of solar radiation would require a distinct magnitude separation at least within the showers whose parent comets are already inactive, and therefore unobservable. Although there are some observations indicating separation of this kind, this is always much less pronounced than the total non-selective dispersion. The drag effects would also require an increase of the mean Jacobian constant with decreasing particle size. However, a comparison of the Prairie Network 
TABLE 3.-Explanations and References to Tables 1 and $\mathscr{Z}$

\begin{tabular}{|c|c|c|c|c|c|}
\hline \multirow{2}{*}{ Type of object } & \multirow{2}{*}{$\begin{array}{c}\text { Mean } \\
\text { absolute } \\
\text { magnitude }\end{array}$} & \multicolumn{3}{|c|}{ Number of objects } & \multirow{2}{*}{ Source } \\
\hline & & Total & $q<1$ & In showers & \\
\hline Numbered asteroids & +11.5 & 1743 & 3 & - & Chebotarev, 1970 \\
\hline Unnumbered asteroids & +17.0 & 10 & 10 & - & Different sources \\
\hline Comets & +6.8 & 607 & 162 & - & $\begin{array}{l}\text { Porter, 1961; Marsden, 1966, } \\
\quad 1970 \mathrm{c}\end{array}$ \\
\hline Super-Schmidt meteors & +1.2 & 359 & 343 & 61 & Hawkins and Southworth, 1961 \\
\hline Bright Super-Schmidt meteors & +0.1 & 413 & 390 & 92 & Jacchia and Whipple, 1961 \\
\hline Small camera meteors & -4.2 & 144 & 143 & 58 & Whipple, 1954 \\
\hline Bright fireballs & -8.5 & 141 & 140 & 8 & McCrosky, 1968, 1970 \\
\hline Brightest fireballs, $H_{E}>30 \mathrm{~km}$ & -12.5 & 16 & 16 & - & \\
\hline Brightest fireballs, $H_{E}<30 \mathrm{~km}$ & -18.0 & 9 & 9 & - & McCrosky, 1970; Ceplecha, 1970 \\
\hline Meteorites & -15.5 & 2 & 2 & - & \\
\hline
\end{tabular}

at $r=1, \Delta=1$ for the asteroids (Gehrels scale) and comets (Vsekhsvyatsky scale); at $\Delta=100 \mathrm{~km}$ for meteors (photographic, maximum light, Harvard scale).

fireballs with the faint Super-Schmidt meteors revealed just the opposite effect.

Attempts were also made to check the presence of librating motions among meteor orbits. These motions seem to be of considerable importance in the evolution of comets and asteroids (Marsden, $1970 \mathrm{~b}$; Kresák, 1972). For meteors there is a slight preference for $3 / 1$ and 41 resonances with Jupiter (Kresák, 1969), but no simultaneous preference for any particular values of the libration argument, which would make this irregularity significant.

\section{FIREBALL END HEIGHTS}

Although the associated problems of meteor physics are beyond the scope of this paper, one correlation of the atmospheric phenomena with the Jacobian constant of the incident particle may be pointed out. Six years of operation of the Prairie Network and tine European Network of all-sky cameras (McCrosky and Ceplecha, 1969) yielded a surprisingly iow gain in the recovery of meteorites compared with the number of brilliant fireballs recorded. This disproportion may point to the existence of two or more types of meteor bodies of different resistances to atmospheric disintegration and possibly, but not necessarily, different types may come from parent bodies of different natures. The parameter for deciding

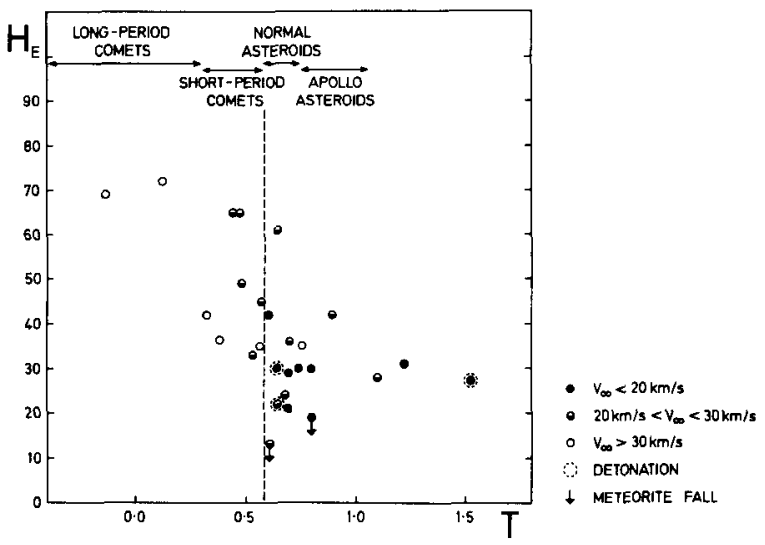

Figure 3.-A plot of meteor end height $H_{E}$ against the Jacobian constant $T$ for very bright fireballs $\left(M_{p}<\right.$ -12). The fireballs are identified by symbols based on the no-atmosphere velocity $V_{\infty}$, and the presence of detonation and meteorite fall. The lower-end heights for asteroidal values of $T(T>0.58$, to the right of the vertical line) is clearly exhibited.

whether we may or may not expect a meteorite fall from a fireball is the end height of the luminous trajectory $H_{E}$. Another important quantity involved both in the mass-brightness dependence and in the resulting end height is the no-atmosphere velocity $V_{\infty}$.

By courtesy of R. E. McCrosky and Z. Ceplecha, detailed data on the brightest fireballs recorded 
by the Prairie Network and the European Network have been obtained. The brightness limit was set at $M_{p}=-12$, and values of the Jacobian constant computed from the orbital elements were plotted against the end heights. The result is shown in figure 3 , with different velocity groups denoted by different symbols to show the possible role of the velocity effect. It is seen that fireballs with a higher extinction level, about $35 \mathrm{~km}$ and more, show a preponderance of cometary values, $T<0.58$, entirely consistent with the situation among faint Super-Schmidt meteors. In contrast to this, a lower extinction level is, without exception, associated with asteroidal values of $T$. This dependence is definitely more pronounced than the obvious relation between $V_{\infty}$ and $H_{E}$. A comparison with the ranges of $T$ occupied by different types of larger objects (fig. 3) suggests that the $H_{E} / T$ dependence may actually be connected with the nature of the parent body; if so, the asteroidal component should prevail slightly over the cometary component at $M_{p}<-12$. However, this assumption would not explain the disproportion between the frequency of bright fireball events and meteorite falls, and nonuniform physical properties within each of the two fundamental groups appear probable.

\section{ACKNOWLEDGMENTS}

The author is greatly indebted to B. G. Marsden, R. E. MoCrosky and Z. Ceplecha for kindly putting at his disposal some of the orbital data used in this paper in advance of publication.

\section{REFERENCES}

Belyaev, N. A., 1967. The orbit evolution of comets Neujmin 2 (1916 II), Comas Sola (1927 III), Schwassmann-Wachmann 2 (1929 I) for 400 years (1660-2060), Astron. Zh., 44, 461-470.

Ceplecha, Z., 1970. private communication.

Chebotarev, G. A., 1970. Ephemerides of minor planets for 1971, Nauka, Leningrad, 166 pp.

Everhart, E., and Raghavan, N., 1970. Changes in total energy of long-period comets, 18001970, Astron. J., 75, 258-272.

Hawkins, G. S., AND Sovthwonth, R. B., 1961. Orbital elements of meteors, Smithson. Contrib. Astrophys., 4, 85-95.

JACCHIA, L. G., AND Whipple, F. L., 1961. Precision orbits of 413 photographic meteors, Smithson. Contrib. Astrophys., 4, 97-129.

Kazimirchak-Polonskaya, H. I., 1967. Evolution des orbites des comètes a courte période au cours des années 1660-2060 et le rôle des planètes extérieures dans cette évolution, Astron. Zh., 44, 439-460.

KRESÁK, L., 1967. Relation of meteor orbits to the orbits of comets and asteroids, Smithson. Contrib. Astrophys., 11, 9-34.

- 1 1968. The relation between orbits and physical characteristics of meteors, in Physics and Dynamics of Meteors, edited by $\check{L}$. Kresák and P. M. Millman, D. Reidel Publ. Co., Dordrecht, Holland, 217-235.

- 1969. The discrimination between cometary and asteroidal meteors. II. The orbits and physical characteristics of meteors, Bull. Astron. Inst. Czech., 20, 231-251.

$\longrightarrow, 1970$. On the orbits of bright fireballs, Bull. Astron. Inst. Czech., 21, 1-9.

- 1972 . On the dividing line between cometary and asteroidal orbits, in The Motion, Evolution if Orbits and Origin of Comets (I.A.U. Symposium No. 45), edited by G. A. Chebotarev, H. T. Kazimirchak-Polonskaya, and B. G. Marsden, D. Reidel Publ. Co., Dordrecht, Holland, 503-514.

Kresák, L̆., ANd PorubČ An, V., 1970. The dispersion of meteors in meteor streams, I. The size of the radiant areas, Bull. Astron. Inst. Czech., 21, 153-170.

Marsden, B. G., 1966. Supplementary catalogue of cometary orbits, Mem. Brit. Astron. Assoc., 40, No. 2, 19 pp.

—, 1968. Comets and nongravitational forces, I, Astron. J., 73, 367-379.

- 1969a. Comets and nongravitational forces, II, Astron. J., 74, 720-734.

—, 1969b. Elements and ephemerides, Minor Planet Circ., Nos. 3014-3016.

- 1970a. Comets and nongravitational forees, III, Astron. J., 75, 75-84.

- 1970b. On the relationship between comets and minor planets, Astron. J., 75, 206-217.

- 1970c. private communication.

—, 1971. 1971 FA, Circ. Bur. Central Telegr. Astron., No. 2320. 
McCrosky, R. E., 1968. Orbits of photographic meteors, in Physics and Dynamics of Meteors, edicted by Ľ. Kresák and P. M. Millman, D. Reidel Publ. Co., Dordrecht, Holland, 265-279.

$\longrightarrow$ 1970. private communication.

McCrosky, R. E., and Ceplecha, Z., 1969. Photographic networks for fireballs, in Meteorite Research, edited by P. M. Millman, D Reidel Publ. Co., Dordrecht, Holland, 600-612.

McCrosky, R. E., AND Posen, A., 1961. Orbital elements of photographic meteors, Smithson. Contrib. Astrophys., 4, 15-84.

Narin, F, ANd Pierce, P. M., 1964. Perturbation, sighting and trajectory analysis for periodic comets, Illinois Research Inst. Astro Sci. Center, Rept. T-7, 107 pp.

ÖPIK, E. J., 1951. Collision probabilities with the planets and the distribution of interplanetary matter, Proc. Roy. Irish Acad., 54A, 165-199.

- , 1963. The stray bodies in the solar system, I. Survival of cometary nuclei and the asteroids, Advan. Astron. Astrophys., 2, 219-262.

Ponter, J. G., 1961. Catalogue of cometary orbits, Mem. Brit. Astron. Assoc., 39, No. 3, 97 pp.

Schubart, J., 1968. Long-period effects in the motion of Hilda-type planets, Astron. J., 73, 99-103.

Southworth, R. B., and Hawkins, G. S., 1963. Statistics of meteor streams, Smithson. Contrib. Astrophys., 7, 261-285.

Stefanik, R. P., 1966. On thirteen split comets, Mém. Soc. Roy. Sci. Liège, 12/1, 29-32.

Van Houten, J. C., Van Houten-Groeneveld, I., Herget, P., and Gehrels, T., 1970. The Palomar-Leiden survey of faint minor planets, Advan. Astron. Astrophys. Suppl., 2, 339-448.

Vsekнsvyatsky, S. K., 1958. Fizicheskie kharakteristiki komet, Gos. Izdatel'stvo fiziko-matematicheskoj literatury, Moskva, $575 \mathrm{pp}$.

Whipple, F. L., 1951. A comet model II. Physical relations for comets and meteors, Astrophys. $J ., 113,464-474$.

- 1954. Photographic meteor orbits and their distribution in space, Astron. J., 59, 201-217.

- 1955. A comet model. III. The zodiacal light, Astrophys. $J$., 121, 750-770.

- 1973. Radial pressure in the solar nebula as affecting the motion of planetesimals, paper no. 38 , this volume. 